\title{
TOPOLOGIES ON THE RATIONAL FIELD
}

\author{
BY M. E. SHANKS ${ }^{1}$ AND SETH WARNER
}

Communicated by R. S. Pierce, June 12, 1973

Although the ring topologies on the field $\boldsymbol{Q}$ of rationals defy classification (see $[2, \S 1]$ ), we are able to resolve a longstanding problem in the theory of topological rings by showing that the only locally bounded ring topologies on $\boldsymbol{Q}$ are the known ones, and in particular, the only Hausdorff, locally bounded, additively generated topology on $\boldsymbol{Q}$ (a ring topology is additively generated if there are no proper open additive subgroups) is the ordinary archimedean topology.

Let $P$ be the set of prime numbers, and for each $p \in P$ let $|\ldots|_{p}$ denote the $p$-adic absolute value on $\boldsymbol{Q}$. Let $|\ldots|_{\infty}$ denote the ordinary archimedean absolute value on $Q$, and let $P^{\prime}=P \cup\{\infty\}$. For each subset $R$ of $P^{\prime}$, let $O(R)=\left\{x \in Q:|x|_{p} \leqq 1\right.$ for all $\left.p \in R\right\}$. As is well known, for each subset $R$ of $P^{\prime}$ there is a unique locally bounded ring topology $\mathscr{T}_{R}$ on $Q$ for which $O(R)$ is a bounded neighborhood of zero (see [1, Exercise 20, pp. 120-121]); if $R \neq P^{\prime}$, a fundamental system of neighborhoods of zero for $\mathscr{T}_{R}$ consists of all $O(R) x$, where $x$ is a nonzero rational. Note that $\mathscr{T}_{P^{\prime}}$ is the discrete topology, and $\mathscr{T}_{\varnothing}$ is the nonHausdorff ring topology.

THEOREM. The only locally bounded ring topologies on $Q$ are the topologies $\mathscr{T}_{R}$, where $R$ is a subset of $P^{\prime}$. In particular, the only Hausdorff, locally bounded, additively generated topology on $\boldsymbol{Q}$ is the ordinary archimedean topology $\mathscr{T}_{\infty}$.

To prove the Theorem, we first identify the completion of $\boldsymbol{Q}$ for $\mathscr{T}_{R}$, where $R$ is a nonempty proper subset of $P^{\prime}$, with the local direct product $A_{R}$ of the fields $Q_{p}$ relative to the open subrings $Z_{p}$, where $p \in R\left(Q_{p}\right.$ and $Z_{p}$ are respectively the field (ring) of $p$-adic numbers (integers) if $p$ is a prime; $\boldsymbol{Q}_{\infty}=\boldsymbol{Z}_{\infty}=$ the real field). The crucial step is to show that if a Hausdorff locally bounded ring topology $\mathscr{T}$ on $\boldsymbol{Q}$ is weaker than $\mathscr{T}_{R}$ for some proper subset $R$ of $P^{\prime}$, then $\mathscr{T}=\mathscr{T}_{S}$ for some proper subset $S$ of $P^{\prime}$; this is accomplished by studying the completion of $Q$ for $\mathscr{T}$ as a topological algebra over the topological ring $A_{R}$. We then apply two results of Mutylin (the only results known thus far concerning the classification of locally bounded ring topologies on $\boldsymbol{Q}$ ); the first [2, Theorem 2] is that if $\mathscr{T}$ is not stronger than $\mathscr{T}_{\infty}$, then $\mathscr{T}=\mathscr{T}_{S}$ for some subset $S$ of $P$ (the above step

AMS (MOS) subject classifications (1970). Primary 12J99, 13J99, 16 A80.

${ }^{1}$ Written while the first author was in residence at Duke University. 
enables us to reduce by half the length of Mutylin's ingenious proof); the second [2, Corollary 5] is that if $\mathscr{T}$ is stronger than $\mathscr{T}_{\infty}$ but not discrete, then $\mathscr{T}$ is weaker than $\mathscr{T}_{S}$ for some proper subset $S$ of $P^{\prime}$.

The following corollaries follow easily; the second generalizes a theorem of Mutylin [2, Theorem 3].

COROLLARY 1. The only nondiscrete locally compact rings containing $\mathbf{Q}$ densely are the rings $A_{R}$, where $R$ is a nonempty proper subset of $P^{\prime}$.

COROLlary 2. If $A$ is a Hausdorff, complete, locally bounded ring containing $\boldsymbol{Q}$, then either $\boldsymbol{Q}$ is discrete, or the closure of $\boldsymbol{Q}$ is $A_{R}$ for some nonempty proper subset $R$ of $P^{\prime}$; in particular, if $A$ is, in addition, a field, then either $\boldsymbol{Q}$ is discrete, or the closure of $\boldsymbol{Q}$ is either the real field or the p-adic number field for some prime $p$.

\section{REFERENCES}

1. N. Bourbaki, Éléments de mathématique. Part 1. Les structures fondamentales de l'analyse. Livre III: Topologie générale. Chaps. III, IV, 3rd ed., Actualités Sci. Indust., no. 916, Hermann, Paris, 1960.

2. A. F. Mutylin, An example of a nontrivial topologization of the field of rational numbers. Complete locally bounded fields, Izv. Akad. Nauk SSSR Ser. Mat. 30 (1966), 873-890. (Russian) MR 33 \#4052.

Department of Mathematics, Purdue University, Lafayette, Indiana 47907

Department of Mathematics, Duke University, Durham, North Carolina 27706 